\title{
Comparison of Plain Radiograph and MRI in Avascular Necrosis of Femoral Head
}

\author{
Banuprakash $\mathbf{S}^{1}$ \\ ${ }^{1}$ Associate Professor, Department of Radio-Diagnosis, Sri Siddhartha Medical College, Agalakote, B H Road, Tumkur- 572107, \\ India
}

Corresponding author: Dr Banuprakash S, Associate Professor, Ths Lab, IMA Building, Town Hall Circle, Tumkur - 572101, India

DOI: http://dx.doi.org/10.21276/ijcmsr.2019.4.1.27

How to cite this article: Banuprakash S. Comparison of plain radiograph and MRI in avascular necrosis of femoral head. International Journal of Contemporary Medicine Surgery and Radiology. 2019;4(1):A108-A110.

\section{A B S T R A C T}

Introduction: Avascular necrosis of the femoral head is a common cause of musculoskeletal disability, and it is a major diagnostic and therapeutic challenge. Though patients are initially asymptomatic, avascular necrosis in majority of cases progresses to joint destruction, clinically manifesting before the $5^{\text {th }}$ decade of life. ${ }^{1}$

OBJECTIVE: To estimate the role of MRI in early evaluation of painful hip joints with subtle plain radiographic findings.

Material and methods: Source of Data: Patients with history of acute or chronic hip pain who have been diagnosed as suffering from AVN by orthopedician are evaluated at to the department of Radio-Diagnosis, Tertiary care centre.

RESULTS:In the present study, Maximum numbers of patients are belongs to age group of $>70$ years and majority of cases are female. Out of 35 cases are diagnosed as AVN of femoral head, only 08 cases are detected on X-Ray but, all 35 cases are detected on MRI.

Conclusion: Avascular necrosis of the head of the femur bone is a common cause of musculoskeletal disability, cascading as a major diagnostic and therapeutic challenge. Though patients are initially asymptomatic, avascular necrosis in majority of cases progresses to joint destruction, clinically manifesting before the $5^{\text {th }}$ decade of life. Femoral head is the most commonly involved site and patients regularly present with pain in hip, sometimes with referred knee pain.

Keywords: AVN, HIP JOINT, MRI, X RAY.

\section{INTRODUCTION}

Avascular necrosis of the femoral head is a common cause of musculoskeletal disability, cascading as a significant diagnostic and therapeutic challenge. Though patients are initially asymptomatic, avascular necrosis in majority of cases progresses to joint destruction, clinically manifesting before the $5^{\text {th }}$ decade of life. ${ }^{1}$

AVN of femoral head denotes ischemic injury of femoral head. The term avascular necrosis is nothing but ischemic necrosis involving areas of epiphyseal or sub articular involvement, whereas infarction of bone usually is reserved for metaphyseal and diaphyseal involvement. The main feature of avascular necrosis is compromised vascular flow leading to osseous cell death. Significant causes include excessive corticosteroid usage, trauma, acute pancreatitis, chronic alcoholism, radiation, sickle cell disease. Femoral head is the most commonly involved site and clinically manifesting at pain in the hip region sometimes with referred knee pain. ${ }^{2}$ For increasing the efficacy of conservative treatment, early detection of femoral head AVN is necessary, with resultant relief from pain and preservation of normal joint function. When subchondral fracture has occurred, the management modalities include a more vigorous treatment such as total hip replacement, with a consequent increase in morbidity.
The sensitivity for detecting early stages of the disease by plain radiograph is as low as $41 \%$. Conventional radiograph is not helpful in detection of stages 0 and 1 of AVN., 3,4

With MRI emerging as the most widely used diagnostic method for avascular necrosis of femoral head, the sensitivity and specificity of diagnosis has been greatly enhanced. Recent research reveals that MRI has a very high sensitivity in diagnosing early lesions based on histology or eventual regression. Early intervention may be possible if asymptomatic, high-risk patients are screened appropriately. Imaging findings are seen as signal-intensity bands or lines within the femoral head are seen surrounding the area that corresponds to ischemic bone on T1- and T2-weighted images. The band is thick on T1-weighted images and is thinner and accompanied by a second, inner band of high signal intensity on T2-weighted images. The double-line sign on T2 weighted images is considered highly specific for AVN. This band is believed to represent the reactive interface that separates normal marrow from infarcted marrow. The signal intensity of the central infarcted bone corresponds to areas of bone necrosis seen at histologic examination. ${ }^{6}$

Viable area of necrosis may display high signal intensity on $\mathrm{T} 1$ weighted images and low signal intensity on T2 weighted images. The accompanying features are presence of fatty marrow with prolonged ischemia and necrosis. The necrotic 
bone shows a signal intensity pattern resembling that of fluid, with low signal intensity and high signal intensity on T1 and T2 weighted images respectively.Fibrosis and sclerosis of the involved bone is reflected by low signal intensity on both T1 and T2 weighted images.MR imaging also helps in detecting secondary signs and course of AVN. Features like joint effusion or cartilaginous thinning may be present. Progression of AVN leads to instability of the femoral head with fragmentation and eventual collapse.

Normal signal intensity of the femoral head or no changes in the signal intensity are seen in the early changes of the diseases. Early sign of AVN is non specific .T1 weighted images with diffuse areas of decreased signal intensity are seen in normally high signal intensity fatty marrow. Although radiography is the primary imaging technique used in the evaluation of patients with suspected or known LCP disease, MRI may play an important complimentary role. Early diagnosis of LCP disease is important because it allows prompt initiation of potentially joint-preserving therapies. MRI depicts the exact extent of femoral head involvement more precisely than pinhole scintigraphs. Study aimed at comparison of plain radiograph and mri in avascular necrosis of femoral head

\section{MATERIAL AND METHODS}

Patients with history of acute or chronic hip pain who have been diagnosed as suffering from AVN by orthopedician were evaluated at the department of Radio-Diagnosis, Tertiary care centre. A cross sectional study was performed and the data was analysed by Proportions with a sample size of 35 cases for a period of one year. 1.5 Tesla Philips Achieva Machine using abdominal surface coils and spine coils were used to take images and Plain radiograph films were taken in all 35 cases. Protocol used for scanning were T1WI and T2 WI spin echo, coronal, sagittal and axial scans of bilateral hip joint were done using body coil and STIR sequences were done in coronal plane.

The criteria used were as following:-

True Positive: Positive AVN changes + indeterminate AVN changes in $\mathrm{X}$-ray confirmed by MR imaging as positive.

True Negative: No changes of AVN in X-ray confirmed as Negative by MR imaging.

False Positive: Positive AVN changes + indeterminate changes in X-ray confirmed by MR imaging as Negative.

False Negative: No changes of AVN in X-ray confirmed by MR imaging as Positive.

Inclusion Criteria: patients presenting with acute or chronic hip pain. Exclusion Criteria: Patients with history of acute trauma.

\section{STATISTICAL ANALYSIS}

The findings of plain radiograph and MRI scan were entered in master chart and software SPSS VERSION 21 has been used to tabulate the above said findings.

\section{RESULTS}

Maximum number of patients are belongs to age group of

\begin{tabular}{|l|c|c|c|}
\hline Age group & Male & Female & Total \\
\hline$<30$ Years & - & - & - \\
\hline 31-40 Years & 01 & 02 & 03 \\
\hline $41-50$ Years & 03 & 05 & 08 \\
\hline $61-70$ Years & 04 & 07 & 11 \\
\hline$>70$ Years & 04 & 09 & 13 \\
\hline Total & 12 & 23 & 35 \\
\hline
\end{tabular}

Table-1: Age and sex wise distribution of cases AVN cases

\begin{tabular}{|l|c|c|}
\hline AVN cases & By X ray & By MRI \\
\hline 35 & 08 & 35 \\
\hline \multicolumn{2}{|c|}{ Table-2: Diagnosis of avn by $\mathrm{X}$ ray and MRI } \\
\hline
\end{tabular}

>7o years and majority of cases are female (table-1).

Out of 35 cases are diagnosed as AVN of femoral head, only 08 cases are detected on X-Ray but, all 35 cases are detected on MRI (table-2).

\section{DISCUSSION}

In the present study, Maximum number of patients are belongs to age group of $>70$ years and majority of cases are female. Out of 35 cases are diagnosed as AVN of femoral head, only 08 cases are detected on X-Ray but, all 35 cases are detected on MRI.

According to a study by B E Vande Berg, the value of high spatial resolution and contrast material-enhanced magnetic resonance (MR) imaging was assessed in 69 patients with either femoral head avascular necrosis (AVN) or transient bone marrow oedema lesions. An AVN lesion was typically a well-demarcated epiphyseal area of variable signal intensity. A transient bone marrow oedema lesion appeared as an ill-delimited low-signal-intensity epiphyseal area on T1weighted images that converted to a high-signal-intensity area on T2-weighted images. T1- and T2-weighted spin-echo images helped distinguish from AVN lesions some transient lesions apt to simulate the segmental pattern of AVN lesions. T2-weighted images also helped detect necrotic tissue in some unusual AVN lesions that mostly showed ill-delimited oedema like marrow changes. In some cases, contrastenhanced MR images may increase diagnostic confidence by showing homogeneous hyper vascularisation in bone marrow oedema lesions and by depicting hypo vascular marrow areas in AVN lesions. The authors believe sequential MR imaging is valuable in the assessment of equivocal femoral head lesions. $^{7}$

According to a study by Ragab, 34 patients complaining of hip pain were assessed.The clinical assessment was done for possible hip disease along with plain radiograph and MRI scan of both hips .31 patients showed unilateral hip involvement(91.2\%),three patient,(8.8\%) shown bilateral hip involvement, with a total of 37 hips evaluated by MRI. ${ }^{8}$

According to a study by Shih, out of 36 hips that were studied with significant hip pain, radiography of the hip showed subtle changes. Single lesion were found in twenty nine hips studied and that included - infection (one case), fracture (eight case), avascular necrosis of the femur (two case), or contra lateral hip (four case), transient osteoporosis (six case), osteoporosis (one case), post-irradiation myositis (one case), 
metastasis (four case), and synovitis (two case). 26(89.6\%) appeared normal on the radiographs of the hip, while three lesions (10.4\%) showed only osteoporotic change. The rest of seven hips showed more than one lesion, the majority being avascular necrosis and fracture (four), fracture foci (two), and metastasis and fracture (two).Hip radiography was not significant in detecting important pathologic foci. MRI shows high degree of sensitivity to alterations in the bone marrow that may denote pathology which may go undetected on plain radiography of hips. Patients with persistent hip pain with no findings on radiography should undergo MRI at the earliest, so that diagnosis and treatment can be planned. ${ }^{9}$

According to a study by Donald G,MR images of documented avascular necrosis (36 hips) and normal hip joint without evidence of joint disease ( 80 hips) were analysed to study the fluid dynamics in the joint. The MRI examinations were done using a $1.5 \mathrm{~T}$ machine which included coronal images of T2 weighting. ${ }^{10}$

According to a study by Khaladkar, 45 patients of AVN femoral head were suspected clinically or on plain $\mathrm{x}$-ray, were subjected for MRI hip. MRI hip was done on $1.5 \mathrm{~T}$ Siemens Avanto. Out of 45 cases, 36 were detected for AVN (total 58 hips), where as MRI did not show AVN findings in nine patients. To start with a limited MRI protocol with coronal T1 weighted images (T1W1) was initiated followed by a complete MRI examination 24.Majority of the patients belonged to age group $31-45(31 \%)$. Majority were males(88.8\%) and females were in minority(11.1\%). AVN was detected in $44(61.1 \%)$ - bilaterally and in 14 patients (38.8\%)-unilaterally. Pre collapsed stage (Stages I and II) was detected in 18 hips (31.03\%), collapsed stage (Stages III and IV) was detected in 40 hips (68.9\%). Peripheral low-intensity rim was seen on T1WI in all 58 cases of AVN. Double line sign on T2-weighted image was seen in 33/58 (56.8\%). The study concluded that coronal T1W sequence is an easy, rapid and cost-effective method of detecting unilateral or bilateral AVN.

\section{CONCLUSION}

Avascular necrosis of the head of the femur bone is increasingly becoming a common cause of musculoskeletal disability with major diagnostic and therapeutic challenges. Most of the patients are initially asymptomatic, avascular necrosis usually progresses to joint destruction, usually before the $5^{\text {th }}$ decade of life. The most commonly involved site is the femoral head and patients regularly present with pain in the lower limbs. The aim of diagnostic imaging procedures in avascular femoral head necrosis is to provide the patient with a stage-adapted therapy. Native radiography of the hip in two planes is still the first step. Over the past years, the diagnosis of femoral head necrosis has experienced tremendous improvement due to the use of MRI. Because of these improvements the correct stage can be diagnosed early and the correct therapy can be started immediately. Now, MRI is the most sensitive diagnostic imaging procedure.

\section{REFERENCES}

1. ZoiaStoica, Daniela Dumitrescu, M. Popescu,
IoanaGheonea, Mihaela Gabor, N. Bogdan Imaging of Avascular Necrosis of Femoral Head: Familiar Methods and Newer Trends Current Health Sciences Journal 2009;35(1).

2. Yochum T, Rowe L. Essentials of skeletal radiology. 2nd ed. Baltimore: Williams \& Wilkins 1996; 260-1263.

3. Resnick D, Niwayama G. Osteonecrosis: diagnostic techniques, special situations and complications. In: Resnick D, ed. Diagnosis of Bone and Joint Disorders. Philadelphia.WB Saunders Co; 1995:3495.

4. Ficat RP, Arlet J. Necrosis of the femoral head. In: Hungerford DS, ed. Ischemia and Necrosis of Bone. Lippincott Williams \& Wilkins;1980:171

5. Steinberg ME, Hayken GD, Steinberg DR. A quantitative system for staging avascular necrosis.J Bone Joint Surg Br. 1995; 77(3):34-41.

6. Bassett, L., et al.: MRI in the Early Diagnosis of Ischemic Necrosis of the Femoral Head. Clin. Ortho. 214:237-248.

7. Vande BergBE, Malghem JJ, Labaisse MA, Noel HM, Maldague BE.MR imaging of avascular necrosis and transient marrow edema of the femoral head. RadioGraphics1993;13(3):4-9.

8. Ragab Y, Emad Y, Abou-Zeid A. Bone marrow oedema syndromes of the hip: MRI features in different hip disorders Clinical rheumatology 10.10.07 10067-0070731.

9. Shih TT, Su CT, Chiu LC, Erickson F, Hang YS, Huang KM. Evaluation of hip disorders by radiography, radionuclide scanning and magnetic resonance imaging. J Formos Med Assoc 1993; 92(8): 737.

10. Donald G. Rao MV, Dalinkal M, Charles E, Spritzer, Jester WB, Axel L. MRI of Joint Fluid in the Normal and Ischemic Hip AJR June 1986; 146(1):1215-1218.

Source of Support: Nil; Conflict of Interest: None

Submitted: 12-01-2019; Accepted: 15-02-2019; Published online: 20-03-2019 